\title{
Implantação e implementação do Apice On: Concepção dos profissionais de saúde
}

\section{Maryelle Peres da Silva Santos', Beatriz Castro Souza Capelanes², Katia Terezinha Alves Rezende ${ }^{3}$ e Mara Quaglio Chirelli ${ }^{3}$}

\author{
${ }^{1}$ Discente de medicina, Faculdade de Medicina de Marília (Famema), Brasil | \\ peresmaryelle@gmail.com | https://orcid.org/0000-0000-0000-0000 \\ 2 Médica, Faculdade de Medicina de Marília (Famema), Brasil | beatrizcscp@gmail.com | \\ https://orcid.org/0000-0000-0000-0000 \\ ${ }^{3}$ Enfermeira, Docente da Faculdade de Medicina de Marilia (Famema), Brasil | \\ katialvesrezende@gmail.com; marachirelli@gmail.com | https://orcid.org/0000-0002-9022- \\ 2680; https://orcid.org/0000-0002-7417-4439
}

Resumo: Introdução: o movimento de humanização do parto e nascimento tem sido caracterizado por resistências. Nesse sentido, é criado o projeto Apice On em hospitais vinculados a instituições de ensino com o objetivo de colaborar com a transformação do modelo tecnocrático no cuidado obstétrico e neonatal e impactar na formação de futuros profissionais. Objetivo: analisar a compreensão dos profissionais de saúde sobre a implantação e implementação do Apice On no cotidiano de um hospital com atividade de ensino e de grande porte do interior do estado de São Paulo, Brasil. Método: pesquisa com abordagem qualitativa, por meio de entrevista com profissionais de saúde e Análise de Conteúdo, Modalidade Temática. Resultados e Discussão: as seguintes categorias foram reveladas: "como realiza o cuidado", "definição de cuidado humanizado", "definição de direito da mulher", "potencialidades para a prática do cuidado humanizado", "desafios para a prática do cuidado humanizado", "estratégias para a prática do cuidado humanizado", "Projeto Apice On" e "protocolos para o cuidado". Assim, elaborou-se o tema "Concepção dos profissionais de saúde sobre o Apice On", dividido em três núcleos de sentido: "Conhecimento sobre o Apice On e identificação de potencialidades"; "Entendimento sobre o Apice On sem identificação de potencialidades"; e "Desconhecimento sobre o Apice On". As diferentes concepções sobre o projeto demonstram desafios em sua implementação. Ao passo que existem avanços na prática do cuidado, como incorporação de práticas baseadas em evidências científicas, existem dificuldades, como a resistência ao novo, infraestrutura inadequada e escassez de profissionais. Conclusão: há falta de coesão entre os profissionais da equipe e a gestão, repercutindo no cuidado e no ensino. Sugere-se ampliar a investigação com gestores e usuárias, utilizando a Análise de Conteúdo, Modalidade Temática.

Palavras-chave: Parto Humanizado; Saúde Materno-infantil; Pesquisa Qualitativa; Hospitais; Apice On.

Deployment and Implementation of Apice On: Conception of Health Professionals

Abstract: Introduction: resistances characterized the movement of humanization of childbirth. The Apice On Project was created with the goal of changing the technocratic model in obstetric and neonatal care, focusing on hospitals linked to educational institutions, and also modifying the training of future professionals. Goal: analyze the comprehension of health professionals about the implantation and implementation of Apice On in the daily life of a largescale teaching hospital in São Paulo's inland city. Method: qualitative approach through interviews with health professionals and Content Analysis, Thematic Mode. Results and Discussion: the following categories were revealed: "how care is carried out", "definition of humanized care", "definition of women's rights", "potential for the practice of humanized care", "challenges to the practice of humanized care", "strategies to the practice of humanized care", "Apice On Project" and "protocols for care". Thus, the theme "Conception of health professionals about Apice On" was elaborated divided into three cores of meaning: "Knowledge of Apice On and identification of potentialities"; " Superficial knowledge of Apice On without identification of potentialities"; and "Lack of knowledge about Apice On". There are challenges in the implementation of the Apice On project, evidenced by the different understandings about it. While there are advances in the practice of care, such as incorporations of practices based on scientific evidence, there are obstacles, such as resistance to the new, inappropriate infrastructure and shortage of professionals. Conclusion: there is a lack of cohesion among staff and management professionals who provide obstetric and neonatal care, reflecting on the teaching and the care itself. It is suggested to expand the investigation to management professionals and patients, utilizing the Content Analysis, Thematic Mode.

Keywords: Humanization of Childbirth; Maternal and Child Health; Qualitative Research; Hospitals; Apice On. 


\section{Introdução}

O modelo tecnocrático tem prevalecido no cuidado obstétrico e neonatal brasileiro, caracterizado pela institucionalização do processo de parir, incorporação acrítica de novas tecnologias, práticas intervencionistas e visão mecanicista, submetendo mulheres a normas e rotinas que se sobrepõem ao seu ritmo fisiológico (Rattner, 2018). Em 1990, tem início uma oposição a esse modelo hegemonicamente praticado no Brasil ao reivindicar pela presença do humanismo na formação ética dos profissionais, pela ciência como princípio da prática e pela inclusão dos desejos e necessidades das pacientes na tomada de decisão dos profissionais de saúde (Bourguignon e Grisotti, 2018; Lokugamage e Pathberiya, 2017).

Assim, surge o movimento pela humanização do parto que tem sido marcado, nacional e internacionalmente por avanços, resistências e retrocessos. A construção da cidadania das mulheres tem um histórico de lutas permeadas pela invisibilização política e civil, uma vez que não são reconhecidas como sujeitos de direitos (Maders e Angelin, 2010). No contexto do cuidado obstétrico e neonatal não é diferente, como assinala Diniz et al (2018) ao afirmar que os movimentos sociais foram fundamentais para a conquista de direitos sexuais e reprodutivos e para a defesa de um processo de parturição fisiológico e individualizado, pautado no respeito às preferências das pacientes juntamente com as indicações obstétricas, conforme preconiza a Organização Mundial de Saúde (2018). A Rede Brasileira pela Humanização do Parto e Nascimento (REHUNA) foi uma das organizações da sociedade civil com papel central na estruturação desse movimento, e, com a parceria do Ministério da Saúde (MS), tornou possível a criação de numerosas iniciativas governamentais na esfera do Sistema Único de Saúde (SUS) por meio de leis, portarias e pactos voltados às mudanças para qualificar a atenção à mulher no ciclo gravídico-puerperal e garantir direitos (Diniz et al, 2018).

Em 2000, o Programa de Humanização no Pré-Natal e Nascimento (PHPN) surge com o objetivo de garantir o acesso ao pré-natal, leitos para o parto e melhor estrutura dos serviços. Em 2011, a Rede Cegonha (RC) é instituída para adequar a atenção ao parto, com gradativa migração para um modelo humanizado (MS, 2011). Entretanto, o modelo tecnocrático ainda persiste nos hospitais, inclusive naqueles com atividade de ensino, nos quais profissionais ali formados reproduzem as vivências e os aprendizados adquiridos de seus professores, reeditando um modelo inadequado. Tal circunstância levou o Ministério da Saúde (MS) e o Ministério da Educação a elaborarem, em 2017, uma proposta de mudança na atenção vigente nesses hospitais: o Projeto de Aprimoramento e Inovação no Cuidado e Ensino em Obstetrícia e Neonatologia (Projeto Apice On), visando impulsionar movimentos de mudança dos modelos tradicionais de formação, atenção e gestão, a partir da implementação e disseminação de práticas fundamentadas pela humanização dos processos de cuidado e pela evidência científica (MS, 2017; Mendes e Rattner, 2020).

Entretanto, há desafios no cumprimento de iniciativas que pretendem assegurar o direito de mulheres e seus bebês. É o que demonstram Capelanes et al. (2020), ao identificar que há falhas na implantação e implementação do projeto Apice On nos hospitais e que elas podem resultar em uma formação, atenção e gestão incapazes de produzir um impacto consistente na educação em saúde, no respeito aos direitos humanos e na defesa da medicina baseada em evidências.

Diante disso, pergunta-se: "como os profissionais de saúde compreendem a implantação e implementação do Apice On, considerando as potencialidades, os desafios e as propostas de superação encontradas durante o processo". Como objetivo, procura-se analisar a compreensão dos profissionais de saúde sobre a implantação e implementação do Apice On no cotidiano de um hospital com atividade de ensino e de grande porte do interior do estado de São Paulo, Brasil. 


\section{Metodologia}

A fim de alcançar o objetivo mencionado acima, optou-se pela abordagem qualitativa para aprofundar a compreensão dos processos, sujeitos, relações sociais e estruturas, que, por sua vez, são depositárias de significados, aspirações, crenças e valores (Minayo, 2014). O trabalho de campo apresentou-se como uma possibilidade de aproximação com o cuidado obstétrico e de criação de conhecimento, partindo da realidade presente em um hospital universitário de grande porte do interior do estado de São Paulo, Brasil. A instituição pesquisada está inserida, desde 2011, na RC e, recentemente, em novembro de 2017, passou a integrar a rede de hospitais que participam do Apice On.

Utilizou-se como critério de inclusão dos participantes trabalhar como profissional de saúde na equipe de referência da unidade de internação e do centro obstétrico durante, no mínimo, seis meses.

Utilizou-se roteiro na entrevista semiestruturada para coletar os dados, sendo composto por caracterização dos participantes e questões norteadoras: "Você conhece o projeto Apice On? Se sim, de que forma?", "Você percebe diferença na prática do cuidado com a implantação e implementação do projeto Apice On? Sem sim, quais? Se não, por quê?".

As entrevistas foram realizadas por duas pesquisadoras. Um piloto foi elaborado para a padronização da estratégia de coleta de dados frente aos disparadores e para a formação das entrevistadoras, com a supervisão de uma orientadora com doutorado.

No dia da entrevista, as pesquisadoras abordaram os profissionais da equipe de referência pertencentes ao serviço de saúde em questão de forma aleatória e no seu local de trabalho, porém em lugar reservado. Mediante a apresentação da pesquisa e da disponibilidade dos profissionais em participar, foi assinado o Termo de Consentimento Livre e Esclarecido (TCLE).

A aprovação da pesquisa ocorreu em 04 de setembro de 2018, pelo Comitê de Ética da instituição pesquisada, sob o parecer de no 2.872 .218 e Certificado de Apresentação para Apreciação Ética (CAAE): 95474418.4.0000.5413. Para manter o sigilo dos participantes e salvaguardar seus nomes, os discursos foram codificados por meio das siglas: ASS médico assistente ginecologista e obstetra; ANE - médico anestesiologista; ATE - auxiliar e técnico de enfermagem; ENF - enfermeiro; RES - médico residente de ginecologia e obstetrícia.

A coleta de dados compreendeu o período de abril a dezembro/2019. Atingiu-se a saturação dos dados com 24 entrevistas, ou seja, com o conteúdo obtido foi possível compreender a lógica interna do grupo de profissionais em relação ao objeto de estudo (Minayo, 2014). Os diálogos, de duração média de 40 minutos, foram gravados, transcritos na íntegra pelas pesquisadoras e validados pela orientadora.

A técnica de análise de dados empregada foi a Análise de Conteúdo, Modalidade Temática, segundo Minayo, Deslandes e Gomes (2016). Para tanto, realizou-se uma préanálise, a qual consistiu em transcrever integralmente as entrevistas, permitindo a impregnação do conteúdo das falas, bem como a retomada dos pressupostos e dos objetivos da pesquisa. Esse momento possibilitou ter uma visão de conjunto, apreender as particularidades e definir um esquema de classificação inicial composto por oito categorias: "como realiza o cuidado", "definição de cuidado humanizado", "definição de direito da mulher", "potencialidades para a prática do cuidado humanizado", "desafios para a prática do cuidado humanizado", "estratégias para a prática do cuidado humanizado", "Projeto Apice On" e "protocolos para o cuidado". A partir disso, explorou-se o material, com distribuição dos trechos das falas e realização de diálogo com as partes de um trecho de uma dada classe. 
Posteriormente, elaborou-se uma descrição dos fragmentos de depoimentos. Por meio de inferência, núcleos de sentido foram identificados e agrupados em temas. Nesse artigo, será apresentado o tema "Concepção dos profissionais de saúde sobre o Apice On", dividido em três núcleos de sentido: "Conhecimento sobre o Apice On e identificação de potencialidades"; "Entendimento sobre o Apice On sem identificação de potencialidades"; e "Desconhecimento sobre o Apice On".

\section{Resultados}

\subsection{Caracterização dos Profissionais de Saúde}

Foram entrevistados 24 profissionais de saúde: 09 médicos ginecologistas e obstetras, 06 auxiliares ou técnicos de enfermagem, 04 médicos residentes de ginecologia e obstetrícia, 03 enfermeiros e 02 médicos anestesiologistas.

\subsection{Apresentação do Tema}

\subsubsection{Concepção dos Profissionais de Saúde sobre o Apice On}

Evidencia-se conhecimento sobre o Apice On e identificação de potencialidades, a partir da participação no Grupo Estratégico Local (GEL), trazendo sua caracterização e relação com a RC.

"Tem reuniões quinzenais do GEL, que é um grupo formado por enfermeiros, médicos do serviço, a enfermeira que representa o Ministério da Saúde e a equipe da DRS da nossa diretoria regional" ENF2.

Em relação às potencialidades, há destaque para a diminuição das cesarianas, episiotomia, prescrição de ocitocina, jejum de rotina e do parto vaginal conduzido em centro cirúrgico, que passou a ser realizado na sala de pré-parto. Assim como, o estímulo à visita da maternidade pela gestante antes do momento do parto, presença do acompanhante durante a internação, visita aberta de familiares, uso de banqueta, bola e exercícios durante o trabalho de parto. Apontou-se também a valorização do desejo das usuárias, incentivo ao aleitamento na primeira hora de vida do recém-nascido e capacitação dos profissionais para inserção do DIU pós-parto - iniciativa que compõe a estratégia de planejamento familiar do projeto.

"Hoje o trabalho de parto é feito com uma equipe humanizada. O marido está do lado, a gente faz o parto no próprio quarto se não tiver nenhuma intercorrência, o RN vai mamar na primeira hora de vida, não vai ser feita episio, a paciente vai se alimentar" ASS3.

Relacionou-se o alto índice de cesáreas à formação médica que vê o processo gravídicopuerperal de forma patológica e não fisiológica. A cesárea pode ser adequadamente indicada e, ainda assim, contar com a presença do acompanhante, do contato pele a pele e do aleitamento na primeira hora após o parto.

"De acordo com as pesquisas, o índice de cesariana no Brasil ainda é muito alto. É um país muito cesarista e pouco se incentiva, estimula e propõe o empoderamento da mulher pro parto vaginal, desmistificando essa questão do medo da dor" ENF2.

"O Apice On também chama atenção porque a hora que você faz uma reunião com todo mundo do hospital e fala 'olha, a sua taxa de cesárea está alta. Taxa de cesárea alta está relacionada com maior mortalidade materna. Nós estamos matando mais mulheres. Então, a gente precisa mudar isso' [...]. Por mais que diretamente não mude a assistência, mas faz você pensar" RES2. 
O Apice On, ao conceber o parto como fisiológico, possibilitou reflexões sobre a prática e impactou na formação dos acadêmicos e residentes, que, futuramente, poderão constituirse como uma geração de profissionais que atue promovendo a integralidade do cuidado

"Ele [Apice On] veio dentro dessas universidades, pra tornar o parto uma coisa mais natural e não instrumentalizado. Então, se você começa a agir assim, quando você se tornar médico, você vai achar que não é mais daquele jeito, então você vai passar para as outras pessoas o jeito que você vai aprender" ASS3.

A transformação do cuidado iniciou-se com os médicos residentes de ginecologia e obstetrícia. Os médicos assistentes formados há mais tempo e inicialmente resistentes a mudanças, refletiram sobre seu cuidado a partir do impacto no cotidiano de trabalho gerado pelo projeto.

"Eu acho que eles [os residentes] são mais novos e se engajaram mais nessa questão do parto vaginal, do parto humanizado. De querer fazer disso uma realidade aqui pro hospital" ASS1.

"A resistência existe pelas tradições,[...]. Mas todas as pessoas tiveram a conscientização da necessidade de mudança e com a visibilização que houve a melhora, as resistências vão diminuindo" ASS3.

No início do Apice On, enfermeiros obstétricos foram inseridos no cotidiano do serviço, mas, posteriormente, foram realocados devido à necessidade de sua presença em outros setores. No período em que atuaram na maternidade, observa-se a tendência em promover o cuidado na direção da integralidade e na realização de maior quantidade de partos vaginais. Ressalta-se a necessidade de aumentar o número de enfermeiros obstétricos e criar protocolos institucionais para regulamentar sua prática.

"Melhorou muito a qualidade da assistência na maternidade com o olhar da enfermeira obstétrica, pois a enfermeira já faz a dinâmica com toque, presta cuidado para o binômio, com olhar diferenciado por conta da especialização de cada uma" ENF1.

Apesar de considerar potencialidades, sinaliza-se limitações no desenvolvimento do projeto, tais como a baixa adesão da equipe, dificuldade na implementação dos quartos PPP (pré-parto, parto e pós-parto) e dos alojamentos conjuntos devido à falta de profissionais e de infraestrutura, comprometendo a privacidade das mulheres e, por consequência, a humanização.

"A gente teve muita pouca adesão dos profissionais, talvez a estratégia de chamamento não foi muito boa. Acho que falta de interesse mesmo." ASS8

"A proposta do Apice On era fazer um leito num quarto com a recepção do RN na mesma sala, tanto de cesárea quanto aqui, e ser individual. Hoje, pela infraestrutura não aconteceu isso ainda, mas isso pra mim é o primordial da humanização" ENF3.

"Os médicos vêm de uma formação muito mais cesarista do que de parto vaginal. São dois leitos só e o trabalho de parto demora. É muito mais resolutivo você fazer uma cesárea do que aguardar um parto vaginal, não só por questões da pessoa, mas por questões do serviço, da lotação" ENF3.

Percebe-se o entendimento sobre o Apice On sem identificação de potencialidades. A informação sobre a existência do projeto ocorreu por conversas informais e na ausência de um espaço institucional destinado a explicar seu significado, metas e competências a serem mobilizadas pela equipe. Revela-se entendimento apenas do nome "Apice On" e de que o projeto ainda não foi implementado, o que é justificado pela falha na gestão em não incluir as equipes envolvidas, provocando insatisfação dos profissionais. 
"Continua a mesma coisa, diz que vai mudar [...]. Por enquanto, ainda não vi mudanças" AET2.

"Já falaram que gostariam de implantar o Apice On, mas não houve nenhuma reunião explicando para todos o que é [...]. Então, a gente sabe o que foi colocado, mas se você não vai pesquisar individualmente isso não é passado de cima pra baixo" ASS6.

"Acho que houve uma falha de quem implantou aqui esse projeto, de não contar com todos os profissionais envolvidos. Quando começa assim, não vai dar certo" ANE1.

"Como você vai colocar o Apice On pra melhorar a qualidade dos partos vaginais aqui se a maioria das equipes nem sabe o que significa o projeto e não teve nenhum treinamento pra ninguém?" RES1.

Nota-se o desconhecimento sobre o Apice On, justificado pela ausência de informação e de capacitação dos profissionais pela gestão do serviço.

"Eu não estou sabendo de nada [sobre o Apice On]. Eu desconheço. Até então, não fomos apresentados. Eu acredito que a maioria não saiba" AET6.

Além do desconhecimento do projeto e de transformações decorrentes dele, aponta-se retrocessos nos últimos anos. Profissionais que presenciaram, antigamente, o local destinado unicamente ao Centro Obstétrico, sem estar associado ao Centro Cirúrgico, não observam avanços recentes. A falta de privacidade é novamente destacada.

"Eu acho que piorou muito, principalmente porque nós tínhamos um centro obstétrico, um lugar específico pra gestante. Hoje juntou tudo no Centro Cirúrgico, ficou tudo misturado" ANE2.

"O que a gente vê [no pré-parto] são dois leitos grudados um no outro em que o marido da outra tá vendo a outra paciente nua parir, gritar. Então, onde é que a gente está vendo algo melhor? Eu não estou" ASS2.

\section{Discussão}

É possível identificar diferenças nas concepções dos profissionais de saúde em relação ao Apice On. Há Conhecimento sobre o Apice On e identificação de potencialidades; Entendimento sobre o Apice On sem identificação de potencialidades; e Desconhecimento sobre o Apice On.

A investigação evidencia que o projeto provocou mudança na prática dos médicos residentes e reflexão dos médicos assistentes, que, no início, apresentavam resistência à mudança, mas, posteriormente, foram sensibilizados a transformar seu trabalho, sob os princípios da humanização. A proposta ministerial de integrar o Apice On à Rede Cegonha, principalmente em hospitais vinculados a instituições de ensino, relaciona-se justamente com a intenção de colaborar com o aprendizado dos futuros profissionais de saúde, para que estes incorporem boas práticas obstétricas e neonatais (MS, 2017). Estratégias de implementação do Apice On enfrentam resistências, pois o cuidado humanizado ainda não foi completamente incorporado pelas equipes, resultando em uma formação, atenção e gestão deficitários em assegurar os direitos das mulheres e a medicina baseada em evidências (Capelanes et al., 2020). O que também foi identificado na presente pesquisa e justificado pela falha na comunicação entre os trabalhadores de saúde e a gestão da instituição.

Muitos hospitais vinculados a instituições de ensino ainda reproduzem práticas tecnocráticas, com altas taxas de cesárea e poucos partos vaginais conduzidos por enfermeiros obstetras (Mendes e Rattner, 2020), constatação observada nas falas de diferentes profissionais entrevistados. Entretanto, destaca-se a tentativa frequente da enfermagem e dos médicos residentes em transformar esse cenário. 
Para tanto, o fortalecimento de enfermeiros obstétricos é uma estratégia de grande impacto para reduzir a violência obstétrica, intervenções desnecessárias e cesáreas sem indicações (Gomes et al., 2020). Observa-se que o projeto Apice On pretende alcançar os mesmos objetivos, mas seu potencial transformador não foi valorizado pela instituição pesquisada, uma vez que até houve tentativa de inseri-los na condução dos partos vaginais, mas devido à deficiência de infraestrutura e de profissionais, a proposta foi descontinuada.

Percebe-se que a infraestrutura é apontada como um importante entrave para a implantação do projeto, o qual possui determinações sobre o espaço físico, como quarto PPP e alojamento conjunto. O espaço físico é importante para garantir a privacidade da mulher, item considerado como retrocesso pelos profissionais entrevistados. Mas, para além dele, a falta de profissionais, a superlotação e a sobrecarga também fundamentam as condições precárias de atenção (Lamy, et al., 2021). Mesmo com as dificuldades mencionadas, é necessário manter esforços para garantir a humanização do parto a partir da coesão das equipes e do desejo coletivo em diminuir prejuízos às pessoas que estão sendo cuidadas (Sanfelice et al., 2020). Nesse sentido, o GEL tem a responsabilidade de inserir nas instituições participantes do projeto as boas práticas absorvidas nos cursos de aprimoramento e realizar seminários regionais para construir coletivamente perspectivas do cuidado obstétrico e neonatal com a comunidade. Tais práticas não prescindem de verbas e, ainda assim, não estão sendo estimuladas.

A reprodução de práticas centralizadoras e excludentes da participação de trabalhadores e usuárias, ou seja, os principais envolvidos na atenção mas que não participam das decisões, acabam construindo um cuidado fragmentado, caracterizado pela hierarquia de poder. Essa organização do processo de trabalho pautada na transmissão de decisões, e não de seu compartilhamento, contribui para ações individualizadas e não coletivas dos trabalhadores de saúde. Logo, faz-se necessário efetivar espaços de gestão compartilhada. (Lamy, et al. 2021; Santos \& Souza, 2021). O mesmo é evidenciado neste estudo, já que a implementação do projeto Apice On não foi feita de uma forma que todos os profissionais de saúde estivessem inseridos nesse processo, tampouco cientes do seu significado e objetivos.

Identifica-se que $o$ ato médico vinculado ao parto e o ensino das práticas aos alunos são colocados à frente dos direitos das mulheres. Portanto, demonstra-se a necessidade de se investir na educação continuada, na formação de profissionais de saúde, divulgação dos direitos das mulheres e institucionalização de protocolos fundamentados na humanização do parto e nascimento (Lamy, et al. 2021).

\section{Conclusão}

A pesquisa qualitativa, por meio da análise temática, possibilitou revelar as concepções dos profissionais de saúde sobre o projeto Apice On e permitiu analisar a implantação e implementação de um projeto proposto pelo governo federal.

O desconhecimento dos profissionais de saúde entrevistados sobre um projeto com início em 2017 demonstra falta de coesão entre os profissionais da equipe obstétrica e neonatal com a gestão. Dessa forma, não apenas haverá discrepâncias em procedimentos e condutas, mas também na aprendizagem de profissionais em formação, um dos focos do projeto.

A apresentação e ampliação do debate junto aos profissionais é fundamental para a apropriação e adesão ao Apice On, demandando que os sujeitos participantes da gestão, atenção e ensino se organizem a partir de uma comunicação horizontal entre os segmentos envolvidos.

Deve partir da gestão a sensibilização dos profissionais sobre o projeto, ressaltando as transformações já ocorridas e estabelecendo metas para alcançar as lacunas encontradas. 
É necessário compreender também como as usuárias do serviço de saúde concebem o cuidado, quais são as expectativas em relação a ele e se possuem conhecimento sobre 0 projeto Apice On.

Identifica-se como limitação a abordagem somente dos profissionais de saúde, sendo necessário ampliar a investigação a partir da visão dos gestores e usuárias.

\section{Referências}

Bourguignon, A. M., \& Grisotti, M. (2018). Concepções sobre humanização do parto e nascimento nas teses e dissertações brasileiras. Saúde e Sociedade, 27(4), 12301245. https://doi.org/10.1590/s0104-12902018170489

Brasil. Ministério da Saúde. Portaria no 1459/GM/MS. (2011, 24 de junho). Implantação da Rede Cegonha.

Brasil. Ministério da Saúde. Secretaria de Atenção à Saúde. Departamento de Ações Programáticas e Estratégicas. (2017). Apice On: Aprimoramento e Inovação no Cuidado e Ensino em Obstetrícia e Neonatologia.

Capelanes, B. C. S., Santos, M. P. da S., Rezende, K. T. A., \& Chirelli, M. Q. (2020). Cuidado humanizado às gestantes, parturientes e puérperas: análise temática da concepção dos profissionais de saúde. New Trends in Qualitative Research, 3, 648-663. https://doi.org/10.36367/ntqr.3.2020.648-663

Diniz, C. S. G., Rattner, D., d'Oliveira, A. P. L., Aguiar, J. M. de, \& Niy, D. Y. (2018). Disrespect and abuse in childbirth in Brazil: social activism, public policies and providers' training. Reproductive Health Matters, 26(53), 19-35. https://doi.org/10.1080/09688080.2018.1502019

Gomes, L. M., Duarte B. A. R., Giuliani, C. D., Ferreira, M. C. M. \& Junqueira, M. A. B. (2020). Violência obstétrica: perspectiva de puérperas atendidas em um hospital universitário no interior de Minas Gerais. Saúde Coletiva, 10(59), 4236-4247

Lamy, Z. C., Gonçalves, L. L. M., Carvalho, R. H. S. B. F., Alves, M. T. S. S. B., Koser, M. E., Martins, M. S., Leal, N. P., \& Thomaz, E. B. A. F. (2021). Atenção ao parto e nascimento em maternidades do Norte e Nordeste brasileiros: percepção de avaliadores da Rede Cegonha. Ciência \& Saúde Coletiva, 26(3), 951-960. https://dx.doi.org/10.1590/141381232021263.26572020

Lokugamage, A. U., \& Pathberiya, S. D. C. (2017). Human rights in childbirth, narratives and restorative justice: a review. Reproductive Health, 14(17). https://doi.org/10.1186/s12978016-0264-3

Maders, A. M. \& Angelin, R. (2010) A construção da equidade nas relações de gênero e o movimento feminista no Brasil: avanços e desafios. https://doi.org/10.15600/22381228/cd.v10n19p91-115

Mendes, Y. M. M. B., \& Rattner, D. (2020). Estrutura e práticas de hospitais integrantes do Projeto Apice ON: estudo de linha de base. Revista de Saúde Pública, 54, 23. https://doi.org/10.11606/s1518-8787.2020054001497

Minayo, M. C. S. (2014). O desafio do conhecimento: pesquisa qualitativa em saúde (14ª ed.). São Paulo: Hucitec.

Minayo, M. C. S., Deslandes, S. F., \& Gomes, R. (2016). Pesquisa social: Teoria, método e criatividade ( $1^{\underline{a}}$ ed.). Rio de Janeiro: Vozes.

Rattner D. (2009) Humanização na atenção a nascimentos e partos: breve referencial teórico. Interface - Comunicação, Saúde, Educação, 13(Suppl. 1), 595-602. https://dx.doi.org/10.1590/S1414-32832009000500011

Sanfelice, C.F.O., Tiburcio, C.A., Anastácio, J.V., \& Barros, G.M. (2020). Improvement course for obstetric nurses of the Apice On Project: an experience report. Escola Anna Nery, 24(2), e20190212. https://doi.org/10.1590/2177-9465-ean-2019-0212

Santos, S. B., \& Souza, K. V. (2021). Rede Cegonha network and the methodological challenges of implementing networks in the SUS. Ciência \& Saúde Coletiva, 26(3), 775-780. https://doi.org/10.1590/1413-81232021263.21462020

World Health Organization. (2018). WHO recommendations: intrapartum care for a positive childbirth experience. 